\title{
Exploiting the Full Potential of $\beta$-Amyloid and Tau PET Imaging for Drug Efficacy Testing
}

\author{
Henryk Barthel ${ }^{1}$, John Seibyl ${ }^{2}$, Adriaan A. Lammertsma ${ }^{3}$, Victor L. Villemagne ${ }^{4,5}$, and Osama Sabri ${ }^{1}$ \\ ${ }^{1}$ Department of Nuclear Medicine, University Hospital Leipzig, Leipzig, Germany; ${ }^{2}$ Institute for Neurodegenerative Disorders, New \\ Haven, Connecticut; ${ }^{3}$ Department of Radiology and Nuclear Medicine, Vrije Universiteit University Medical Center, Amsterdam, The \\ Netherlands; ${ }^{4}$ Department of Molecular Imaging and Therapy, Austin Health, Heidelberg, Australia; and ${ }^{5}$ Department of Medicine, \\ University of Melbourne, Heidelberg, Australia
}

$\mathbf{T}$ he feasibility of acquiring clinically relevant histopathologic information on critical underlying amyloid and tau pathology in Alzheimer disease and related diseases is the reason why therapeutic trials now incorporate PET imaging biomarkers in most studies. Specifically, PET is used to assess eligibility for inclusion in trials, as $15 \%-30 \%$ of clinically diagnosed Alzheimer disease participants may have no demonstrable brain amyloid or tau deposition, the very targets of treatment. Furthermore, PET imaging is used to longitudinally monitor both disease-related changes in Alzheimer disease brains and the effects of a drug on the biologic target of interest (1).

Although longitudinal studies require that the PET signal have both high precision and quantitative accuracy, there is a vigorous ongoing debate on how these PET studies should be performed and what kind of PET measures should be generated. This debate is based on the fact that PET is capable of deriving - depending on the data acquisition and analysis protocols used-a wide spectrum of quantitative measures of different quality. This spectrum ranges from semiquantitative tissue ratios (a static measure at a certain time after tracer administration relative to a reference region) such as SUV ratios (SUVRs) to fully quantitative data on the regional concentration of amyloid or tau aggregates, which can be expressed as binding potentials, distribution volumes, distribution volume ratios, or rate constants. The latter fully quantitative data are derived from dynamic brain scans, using either noninvasive (reference region) or invasive (arterial blood sampling) tracer input information, and by applying pharmacokinetic compartmental models.

To decide on the type of quantitative amyloid or tau PET parameters to be used for drug efficacy testing, different aspects need to be considered. On the one hand, one may advocate for fully validated quantification (using a dynamic scan protocol together with tracer kinetic modeling) as the optimal measure of amyloid or tau for assessing drug effects. On the other hand, on the basis of both patient comfort and scanning costs, a short static scan protocol may be attractive. Finally, there is the desire of pharmaceutical companies to optimize the cost-to-benefit ratio of trials and to acquire scientifically strong and regulatory robust data

Received Apr. 20, 2020; revision accepted Apr. 29, 2020.

For correspondence or reprints contact: Henryk Barthel, Department of Nuclear Medicine, University Hospital Leipzig, Liebigstrasse 18, 04103 Leipzig, Germany.

E-mail: henryk.barthel@medizin.uni-leipzig.de

Published online May 15, 2020.

COPYRIGHT (c) 2020 by the Society of Nuclear Medicine and Molecular Imaging. DOI: 10.2967/jnumed.119.228346 that can support a potential filing for approval. These different perspectives require a compromise in order to maximize the robustness and reliability of the outcome data while minimizing discomfort to the patient and costs. So far, in most studies, longitudinal SUVRs have been used to determine pathologic burden at baseline and after treatment. Here, we (an international group of amyloid and tau PET imaging and PET quantification experts) would like to provide a critical appraisal of this approach and present some arguments on how to achieve a compromise that ensures robust, reliable, and reproducible measures of pathologic burden in the brain.

The SUVR has the seductive advantage of being simple to determine from a static short (15-20 min) scan. Consequently, SUVR is less sensitive to head movements and indeed allows for higher patient throughput-that is, reduced scanning costs. Nevertheless, important drawbacks are the lack of an appropriate input function (i.e., delivery to tissues); the sensitivity of SUVR regarding the scanning time window used (because the tracer might not reach apparent steady state, as happens with some amyloid and most tau PET tracers); and the inability to correct for confounding effects (e.g., it is not possible to distinguish between specific and nonspecific uptake). As a result, SUVRs are vulnerable to changes in blood flow affecting tracer delivery and clearance, changes that can be induced by the treatment drug itself, by the response to treatment, or by progression of disease. Furthermore, the lack of an input function precludes accounting for the likely drug-induced changes that might affect tracer delivery to the brain, such as changes in tracer metabolism, blood-brain barrier permeability, or peripheral binding. Investigating this issue, van Berckel et al. found that changes in ${ }^{11} \mathrm{C}$-Pittsburgh compound $\mathrm{B}$ SUVR over time differed from those parameters derived from the gold standard kinetic modeling. The discrepancy was attributed to longitudinal changes in blood flow (2). Although this discrepancy may not have a strong effect in healthy elderly controls, brain perfusion progressively becomes impaired in dementia patients, adding to concerns about the use of SUVRs in longitudinal amyloid and especially tau PET imaging, for which reductions in SUVR may be due to changes in these confounding effects rather than real changes in brain target density.

Semiquantitative and even visual (qualitative) methods are valid techniques to demonstrate brain amyloid or tau deposition, which can serve as an inclusion criterion for clinical trials. Nevertheless, given the confounding effects mentioned above, it is strange that the same methods have subsequently also been used in longitudinal studies without critical assessment of their validity. We strongly believe that these simplified methods can be used in longitudinal 


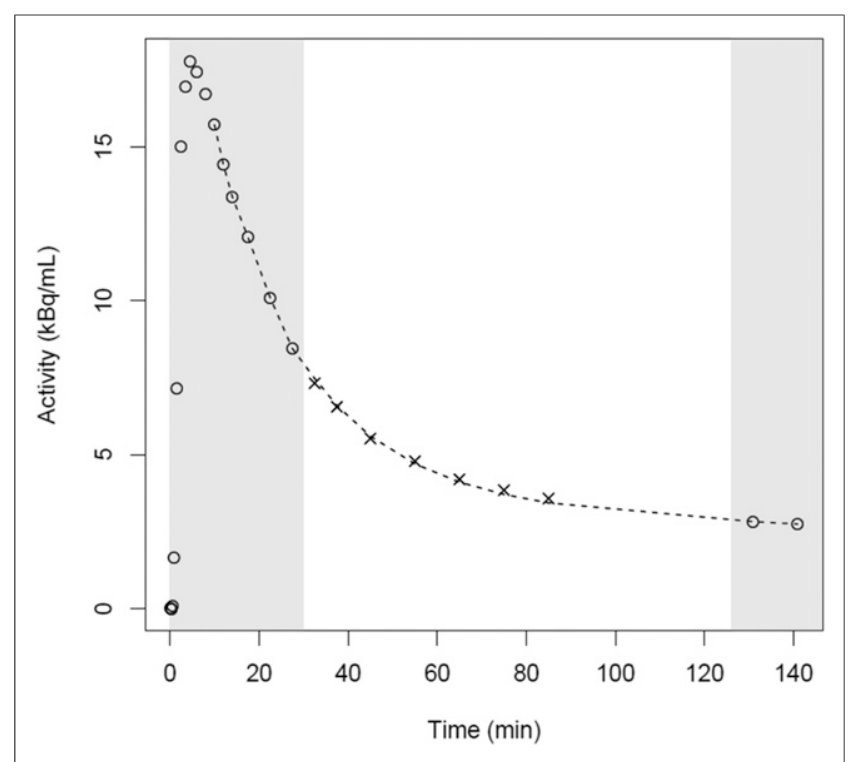

FIGURE 1. Example of so-called coffee break or dual-time-window imaging protocol, in this case for ${ }^{18} \mathrm{~F}$-florbetaben amyloid PET scan (6). Gray-shadowed time windows represent significantly shortened (as compared with full dynamic imaging) scan times for patients, whereas data in in-between interval are interpolated. This protocol increases patient compliance while still providing full-quality quantitative data. It even allows for high patient throughput via interleaved patient scheduling on PET scanner.

studies only after proper validation against corresponding gold standard kinetic modeling measures. In principle, this validation needs to be repeated for each new drug, as confounding effects may be drug-dependent. This validation should also include determination of test-retest variability for the various PET parameters. Only then is it possible to determine whether a simplified approach can be used. In addition, this validation allows for an appropriate power calculation concerning the study cohort size required to answer the trial question and, consequently, to come to a scientifically reasonable and ethically justifiable study design (3). Of note in this regard, the Amyloid Imaging to Prevent Alzheimer's Disease modeling group recently discovered that the use of distribution volume ratios can, as compared with SUVRs, reduce the sample size needed to detect longitudinal amyloid changes by around $40 \%$ in amyloid-negative populations (4), suggesting that distribution volume ratios are much more sensitive than SUVRs in detecting small amyloid changes over time. These results validate the approach and make it even more economically attractive to pharmaceutical industries, which are sometimes risking significant financial resources on unreliable outcome measures. Proper amyloid or tau PET drug efficacy testing protocols would at least shield against valid criticism of some trial reports, as were occasionally published in the past (5).

In therapeutic trials, as a viable alternative to static SUVRs to report on amyloid or tau PET, or when it is not possible to perform fully dynamic imaging for kinetic modeling, dual-time-window protocols should be used (Fig. 1) (6,7). Here, depending on the tracer used, it is necessary to acquire PET data only early after tracer administration and again at a later time point, after a "coffee break." The missing data on the in-between tracer dynamics are then interpolated from the information measured at the 2 time windows. This approach allows for full quantification, including determination of a noninvasive reference-tissue input function. At the same time, the overall length of the scan can be reduced significantly, thus minimizing patient discomfort. This approach is logistically feasible for most dementia patients while still providing robust, high-quality, quantitative drug-efficacy outcome measures and with the potential for higher patient throughput on the PET scanner than is possible with full dynamic scans.

\section{DISCLOSURE}

John Seibyl works as a consultant to Biogen and holds an equity stake in Invicro. Victor Villemagne received the following grant support for related research: NIH RF1 AG053550-01 and NHMRC GA16788. His financial relationships outside the presented work include personal fees for advice and speaker honoraria from Roche, IXICO, Lundbeck, Shanghai Green Valley, Avid Radiopharmaceuticals, Piramal Imaging, and GE Healthcare. No other potential conflict of interest relevant to this article was reported.

\section{REFERENCES}

1. Barthel H, Seibyl J, Sabri O. The role of positron emission tomography imaging in understanding Alzheimer's disease. Expert Rev Neurother. 2015;15:395-406.

2. van Berckel BN, Ossenkoppele R, Tolboom N, et al. Longitudinal amyloid imaging using ${ }^{11} \mathrm{C}-\mathrm{PiB}$ : methodologic considerations. J Nucl Med. 2013;54:15701576.

3. Lammertsma AA. Forward to the past: the case for quantitative PET imaging. $J$ Nucl Med. 2017;58:1019-1024.

4. Lopes Alves I, Collij L, Heeman F, et al. Reducing sample sizes to detect longitudinal amyloid accumulation. Presented at: Human Amyloid Imaging Conference; Miami, Florida; January 17, 2020.

5. Sevigny $\mathrm{J}$, Chiao $\mathrm{P}$, Bussière $\mathrm{T}$, et al. Addendum: the antibody aducanumab reduces A $\beta$ plaques in Alzheimer's disease. Nature. 2017;546:564

6. Bullich S, Barthel H, Koglin N, et al. Validation of noninvasive tracer kinetic analysis of ${ }^{18} \mathrm{~F}$-florbetaben PET using a dual-time-window acquisition protocol. J Nucl Med. 2018;59:1104-1110.

7. Heeman F, Yaqub M, Lopes Alves I, et al. Optimized dual-time-window protocols for quantitative $\left[{ }^{18} \mathrm{~F}\right]$ flutemetamol and $\left[{ }^{18} \mathrm{~F}\right]$ florbetaben PET studies. EJNMMI Res. 2019;9:32. 